\title{
Saúde do trabalhador em Unidades de Terapia Intensiva: Tendências da produção científica brasileira
}

\author{
Worker health in Intensive Care Units: Trends in brazilian scientific production \\ Salud del trabajador en Unidades de Cuidados Intensivos: Tendencias de la producción científica \\ brasileña
}

Recebido: 13/04/2021 | Revisado: 20/04/2021 | Aceito: 22/04/2021 | Publicado: 07/05/2021

\author{
Elisa Gomes Nazario \\ ORCID: https://orcid.org/0000-0001-6129-5218 \\ Universidade Federal de Santa Maria, Brasil \\ E-mail: nazario.elisa@gmail.com \\ Rosângela Marion da Silva \\ ORCID: https://orcid.org/0000-0003-3978-9654 \\ Universidade Federal de Santa Maria, Brasil \\ E-mail: cucasma@terra.com.br \\ Getúlio Simões Nicoletti \\ ORCID: https://orcid.org/0000-0001-8123-4535 \\ Universidade Franciscana, Brasi \\ E-mail: getulio.sn@hotmail.com
}

\begin{abstract}
Resumo
O objetivo do estudo foi identificar as tendências da produção científica, em teses e dissertações, sobre saúde do trabalhador em unidades de terapia intensiva. Trata-se de uma revisão do tipo narrativa. O levantamento das produções ocorreu no mês de maio de 2020 com busca online no Catálogo de Teses e Dissertações da Coordenação de Aperfeiçoamento de Pessoal de Nível Superior (CAPES) utilizando as palavras "saúde do trabalhador" AND "terapia intensiva". Após a aplicação de critérios de seleção, foram abarcadas para análise 36 produções. Nos resultados foi possível identificar que a maior parte das produções foram dissertações de cursos de mestrado em enfermagem, da região sudeste do Brasil e após o ano de 2012. As pesquisas foram predominantemente realizadas com trabalhadores de enfermagem, abrangendo as UTI neonatal, pediátrica e adulta. Nas temáticas destacadas estão as características próprias do trabalho em terapia intensiva e das profissões da área da saúde, bem como os riscos ocupacionais que estão presentes no cotidiano das UTI. Nas considerações finais enfatizamos que nas produções analisadas ficou evidente a constante e importante relação entre a influência das atividades laborais e a saúde dos trabalhadores de unidades de terapia intensiva.
\end{abstract}

Palavras-chave: Saúde do trabalhador; Unidades de Terapia Intensiva; Enfermagem.

\begin{abstract}
The aim of the study was to identify trends in scientific production, in theses and dissertations, on occupational health in intensive care units. This is a narrative-type review. The survey of productions took place in May 2020 with an online search in the Catalog of Theses and Dissertations of the Coordination for the Improvement of Higher Education Personnel (CAPES) using the words "worker health" AND "intensive therapy". After the application of selection criteria, a total of 36 productions were included for analysis. In the results, it was possible to identify that most of the productions were dissertations from master's courses in nursing, from the southeastern region of Brazil and after the year 2012. The studies were predominantly used with nursing workers, including neonatal, pediatric and adult ICUs. The highlighted themes are characteristic of intensive care work and health professions, as well as the occupational risks that are present in the daily lives of ICUs. In the final remarks, we emphasize that in the analyzed productions, the constant and important relationship between the influence of work activities and the health of workers in intensive care units was evident.
\end{abstract}

Keywords: Occupational health; Intensive Care Units; Nursing.

\section{Resumen}

El objetivo fue identificar tendencias en la producción científica, en tesis y disertaciones, sobre salud ocupacional en unidades de cuidados intensivos. Esta es una revisión de tipo narrativo. La elección de producciones se realizó en mayo de 2020 con una búsqueda en el Catálogo de Tesis y Disertaciones de la Coordinación para la Perfeccionamiento del Personal de Educación Superior (CAPES) utilizando las palabras "salud del trabajador" Y "cuidados intensivos". Con aplicación de los criterios de selección, se incluyeron 36 producciones para su análisis. En los resultados se pudo identificar que la mayoría de las producciones fueron disertaciones de maestrías en enfermería, 
de la región sureste de Brasil y posteriores al año 2012. Las investigaciones se realizaron con trabajadores de enfermería, abarcando unidades neonatal, pediátrica y adulta. Los temas destacados incluyen las características del trabajo de cuidados intensivos y las profesiones de la salud, así como los riesgos laborales que están presentes en la vida diaria de las UCI. En las observaciones finales, destacamos que en las producciones analizadas se evidenció la constante e importante relación entre la influencia de las actividades laborales y la salud de los trabajadores en las unidades de cuidados intensivos.

Palabras clave: Salud laboral; Unidades de Cuidados Intensivos; Enfermería.

\section{Introdução}

A relação entre trabalho e saúde é complexa, e esse fenômeno é permeado por diversos fatores que podem influenciar em agravos ao trabalhador (Camargo, 2017). Nessa perspectiva, existe a necessidade de assegurar apropriadas condições para o desenvolvimento das atividades dos trabalhadores, protegendo e promovendo sua saúde (Forte, et al, 2014).

Desse modo, a atenção a saúde do trabalhador deve ser uma questão amplamente discutida. Ela pode ser definida como uma dimensão de práticas e de conhecimentos que busca compreender as associações entre o trabalho e a saúde, e intervém nesse processo por meio de ações de promoção e proteção do trabalhador no ambiente laboral (Gomez, Vasconcellos, Machado, 2018).

Quando se pensa nos trabalhadores de saúde, considera-se que existem diversas situações rotineiramente vivenciadas que podem gerar implicações para manutenção de seu bem-estar (Ribeiro, et al, 2016). Torna-se essencial que exista a promoção de condições adequadas de trabalho e que a saúde ocupacional seja promovida (Forte, et al, 2014).

No contexto das Unidades de Terapia Intensiva (UTI), que se diferenciam no ambiente hospitalar pelas características de alta complexidade de cuidados, uso de tecnologias avançadas, e pacientes em estado grave (Santos, et al. 2018), há ainda mais exigências quanto a atuação dos trabalhadores de saúde que desenvolvem suas atividades nesses locais (Silva, 2019).

Considerando essas questões, se torna relevante a reflexão sobre aspectos relacionados a saúde do trabalhador, especificamente no que se refere as condições nas quais são desenvolvidas as atividades laborais nos setores de terapia intensiva e suas repercussões para os trabalhadores.

Assim, para guiar o atual trabalho, utilizou-se a questão problematizadora: "quais as tendências da produção científica, em teses e dissertações, sobre saúde do trabalhador em unidades de terapia intensiva?". E o seu objetivo foi identificar as tendências da produção científica, em teses e dissertações, sobre saúde do trabalhador em unidades de terapia intensiva.

\section{Metodologia}

O presente estudo é uma revisão do tipo narrativa. Esta forma possibilita a investigação de temáticas abrangentes, é indicada para se obter um levantamento de produções científicas disponíveis e permite a reunião de materiais de diferentes fontes para análise ampla e crítica (Lacerda \& Costenaro, 2016). Nesse sentido, a revisão narrativa torna possível conhecer o estado da arte de determinada temática, contribuindo para fundamentar estudos científicos teoricamente, além de elucidar possíveis lacunas do conhecimento da área investigada (ibidem).

O levantamento das produções foi realizado no mês de maio de 2020 por meio da busca online no Catálogo de Teses e Dissertações da Coordenação de Aperfeiçoamento de Pessoal de Nível Superior (CAPES). Como estratégia de busca foram utilizadas as palavras "saúde do trabalhador" AND "terapia intensiva", obtendo-se 68 produções naquele momento.

Os critérios de seleção foram: resumos completos das dissertações e teses, disponíveis online, produzidas até o ano de 2018, que fossem da temática e abordassem no título ou no resumo aspectos da saúde do trabalhador em unidades de terapia intensiva. Após a aplicação dos critérios, foram selecionadas para análise 36 produções. A partir dos dados coletados nos resumos foram identificadas algumas características das tendências das produções, que são detalhadamente descritas a seguir. 


\section{Resultados}

Das 36 produções encontradas, três foram teses e 33 foram dissertações. Para melhor apresentação dos dados e visualização das produções foi construído um quadro incluindo um código identificador de cada estudo selecionado (sendo T1, T2, para teses e D1, D2 para dissertações), sua referência e também os objetivos (Quadro 1).

Quadro 1: Apresentação das produções selecionadas. Santa Maria, RS, 2020.

\begin{tabular}{|c|c|c|}
\hline $\mathbf{N}^{\mathbf{0}}$ & REFERÊNCIA & OBJETIVOS \\
\hline D1 & $\begin{array}{c}\text { Petersen, R. S. (2012). Fatores do trabalho associados à lombalgia } \\
\text { não especificada, caracterizada no âmbito da resistência da } \\
\text { musculatura extensora lombar, entre trabalhadores de enfermagem } \\
\text { de Unidades de Terapia Intensiva. } 141 \text { p. Dissertação (Mestrado em } \\
\text { Enfermagem Fundamental) - Universidade de São Paulo/ Ribeirão } \\
\text { Preto, Ribeirão Preto. }\end{array}$ & $\begin{array}{l}\text { Identificar os fatores do trabalho associados à lombalgia não específica, } \\
\text { caracterizada no âmbito da resistência da musculatura extensora lombar, } \\
\text { em trabalhadoras de enfermagem de Unidades de Terapia Intensiva. }\end{array}$ \\
\hline D2 & $\begin{array}{c}\text { Corrêa, C. F. (2006). Biossegurança em uma unidade de terapia } \\
\text { intensiva: a percepção da equipe de enfermagem. } 99 \text { p. Dissertação } \\
\text { (Mestrado em Enfermagem) -Universidade Federal do Rio de Janeiro, } \\
\text { Rio de Janeiro. }\end{array}$ & $\begin{array}{l}\text { Descrever as medidas de biossegurança adotadas pela equipe de } \\
\text { enfermagem durante a assistência prestada em UTI; identificar a } \\
\text { percepção da equipe de enfermagem acerca da importância da adoção e } \\
\text { implementação das medidas de biossegurança durante a assistência } \\
\text { prestada em UTI; analisar as possibilidades de implementação, pela } \\
\text { equipe de enfermagem, de medidas de biossegurança durante a } \\
\text { assistência prestada em UTI. }\end{array}$ \\
\hline D3 & $\begin{array}{l}\text { Campos, J. F. (2008). Trabalho em terapia intensiva: avaliação dos } \\
\text { riscos para a saúde do enfermeiro. } 120 \text { p. Dissertação (Mestrado em } \\
\text { Enfermagem) -Universidade do Estado do Rio de Janeiro, Rio de } \\
\text { Janeiro. }\end{array}$ & $\begin{array}{l}\text { Mensurar e avaliar os riscos de adoecimento relacionados ao trabalho do } \\
\text { enfermeiro de UTI a partir do Inventário sobre Trabalho e Riscos de } \\
\text { Adoecimento (ITRA). }\end{array}$ \\
\hline D4 & $\begin{array}{c}\text { Faria, A. M. (2016). Presenteísmo: fatores envolvidos no processo } \\
\text { decisório de ir trabalhar doente. } 175 \text { p. Dissertação (Mestrado em } \\
\text { Trabalho, Saúde e Ambiente) -Fundação Jorge Duprat Figueiredo de } \\
\text { Segurança e Medicina do Trabalho, São Paulo. }\end{array}$ & $\begin{array}{l}\text { Verificar a existência de presenteísmo e os principais fatores envolvidos } \\
\text { no processo decisório de ir trabalhar doente em uma Unidade de Terapia } \\
\text { Intensiva Neonatal (UTIN). }\end{array}$ \\
\hline D5 & $\begin{array}{l}\text { Silva, A. F. (2017). Transtornos mentais comuns, absenteísmo e } \\
\text { presenteísmo na equipe multiprofissional de saúde em uma Unidade } \\
\text { de Terapia Intensiva. } 145 \text { p. Dissertação (Mestrado em Enfermagem } \\
\text { Fundamental) -Universidade de São Paulo, Ribeirão Preto, São Paulo. }\end{array}$ & $\begin{array}{c}\text { Relacionar as características sociodemográficas, laborais e de saúde dos } \\
\text { membros da equipe multiprofissional da Unidade de Terapia Intensiva } \\
\text { (UTI), com a presença de Transtornos Mentais Comuns e a ocorrência de } \\
\text { absenteísmo e presenteísmo. }\end{array}$ \\
\hline D6 & $\begin{array}{c}\text { Oliveira, M. M. (2005). Alterações psicofisiológicas dos } \\
\text { trabalhadores de enfermagem no serviço noturno. } 127 \text { p. Dissertação } \\
\text { (Mestrado em Enfermagem) -Universidade Federal do Rio de Janeiro, } \\
\text { Rio de Janeiro. }\end{array}$ & $\begin{array}{l}\text { Identificar as alterações psicofisiológicas nos trabalhadores de } \\
\text { enfermagem do serviço noturno que atuam em um Centro de Terapia } \\
\text { Intensiva (CTI); descrever as repercussões que as alterações } \\
\text { psicofisiológicas trazem para a saúde do trabalhador de enfermagem do } \\
\text { serviço noturno que atua em um CTI; discutir as estratégias defensivas } \\
\text { utilizadas pelo trabalhador de enfermagem do serviço noturno para lidar } \\
\text { com as alterações psicofisiológicas geradas pelo serviço noturno. }\end{array}$ \\
\hline T1 & $\begin{array}{l}\text { Louro, T. Q. (2015). O adoecimento do profissional de enfermagem } \\
\text { em UTI: o ruído ambiental como marcador de uma síndrome. } 125 \text { p. } \\
\text { Tese (Doutorado em Enfermagem e Biociências) -Universidade } \\
\text { Federal do Estado do Rio De Janeiro, Rio de Janeiro. }\end{array}$ & $\begin{array}{l}\text { Avaliar a repercussão do ruído ambiental nos profissionais de } \\
\text { enfermagem atuantes em UTIs; quantificar o nível do ruído ambiental } \\
\text { encontrado nas UTIs; quantificar o tempo estímulo-resposta do } \\
\text { profissional de enfermagem aos equipamentos utilizados na assistência } \\
\text { aos clientes nas UTIs; defender a Tese de que os ruídos ambientais têm } \\
\text { repercussão no corpo dos profissionais e contribuem para os riscos na } \\
\text { atenção e no cuidado dos clientes. }\end{array}$ \\
\hline D7 & $\begin{array}{c}\text { Campelo, T. L. (2017). O trabalho como operador de saúde na visão } \\
\text { da ergonomia da atividade: estudo de caso da atividade de trabalho } \\
\text { de uma equipe de enfermagem em unidades de terapia intensiva } \\
\text { neonatal e pediátrica. } 170 \text { p. Dissertação (Mestrado em Trabalho, } \\
\text { Saúde e Ambiente) -Fundação Jorge Duprat Figueiredo de Segurança } \\
\text { e Medicina do Trabalho, São Paulo. }\end{array}$ & $\begin{array}{l}\text { Compreender a relação entre o trabalho e o processo saúde-doença de } \\
\text { uma equipe de enfermagem em Unidade de Terapia Intensiva Neonatal } \\
\text { (UTIN) e na Unidade de Terapia Intensiva Pediátrica (UTIP) em um } \\
\text { hospital público, evidenciando as condições de trabalho e os fatores } \\
\text { organizacionais que limitam a margem de ação dos trabalhadores na } \\
\text { regulação de suas atividades. }\end{array}$ \\
\hline D8 & $\begin{array}{c}\text { Macedo, A. P. F. O. (2013). Morbidade referida pelos trabalhadores } \\
\text { de enfermagem na unidade de terapia intensiva. } 74 \text { p. Dissertação } \\
\text { (Mestrado em Enfermagem) -Universidade Universus Veritas } \\
\text { Guarulhos, Guarulhos. }\end{array}$ & $\begin{array}{l}\text { Analisar a morbidade referida pelos trabalhadores de enfermagem na } \\
\text { Unidade de Terapia Intensiva (UTI) e identificar o tratamento utilizado } \\
\text { pelos trabalhadores de enfermagem frente à morbidade referida. }\end{array}$ \\
\hline D9 & $\begin{array}{c}\text { Tyll, M. A. G. (2014). A vivência do estresse profissional na unidade } \\
\text { de terapia intensiva: vozes de profissionais de saúde no Pará. } 89 \text { p. } \\
\text { Dissertação (Mestrado em Ciências Ambientais e Saúde) -Pontifícia } \\
\text { Universidade Católica de Goiás, Goiânia. }\end{array}$ & $\begin{array}{l}\text { Compreender a vivência do estresse profissional nas Unidades de Terapia } \\
\text { Intensiva de um Hospital de referência, para identificar os principais } \\
\text { fatores que contribuem ao estresse ocupacional e identificar o impacto } \\
\text { subjetivo das fontes de estresse sobre o profissional e seu trabalho. }\end{array}$ \\
\hline D10 & $\begin{array}{c}\text { Rennó, C. O. (2012). Análise postural da equipe de enfermagem } \\
\text { durante o banho no recém-nascido. } 90 \text { p. Dissertação (Mestrado em } \\
\text { Enfermagem) -Universidade Federal do Estado do Rio de Janeiro, Rio } \\
\text { de Janeiro. }\end{array}$ & $\begin{array}{c}\text { Identificar as posturas predominantes adotadas pelos trabalhadores de } \\
\text { enfermagem durante o banho no recém-nascido em incubadora neonatal; } \\
\text { Discutir os possíveis riscos para os trabalhadores de enfermagem em } \\
\text { decorrência das posturas adotadas; Analisar as implicações das posturas } \\
\text { para os trabalhadores de enfermagem. }\end{array}$ \\
\hline $\mathbf{T} 2$ & $\begin{array}{c}\text { Azambuja, E. P. (2007). É possível produzir saúde no trabalho da } \\
\text { enfermagem? Um estudo sobre as relações existentes entre a } \\
\text { subjetividade do trabalhador e a objetividade do trabalho. } 276 \text { p. } \\
\text { Tese (Doutorado em Enfermagem) - Universidade Federal de Santa } \\
\text { Catarina, Florianópolis. }\end{array}$ & $\begin{array}{l}\text { Compreender, partindo das crenças, dos valores e das percepções } \\
\text { verbalizados pelos trabalhadores de enfermagem, que ações } \\
\text { desenvolvidas por eles potencializam a sua saúde ou o seu desgaste; e, } \\
\text { segundo, identificar as possibilidades (ou impossibilidades) de expressão } \\
\text { da subjetividade dos trabalhadores da enfermagem, bem como as ações } \\
\text { que se aproximam da produção da saúde ou que são potencializadoras do }\end{array}$ \\
\hline
\end{tabular}




\begin{tabular}{|c|c|c|}
\hline & & $\begin{array}{c}\text { desgaste, por meio da observação das suas ações no âmbito institucional e } \\
\text { organizacional. }\end{array}$ \\
\hline D11 & $\begin{array}{l}\text { Leite, I. R. L. (2012). Carga de trabalho de enfermagem em unidade } \\
\text { de terapia intensiva e fatores associados à saúde do trabalhador. } 91 \\
\text { p. Dissertação (Mestrado em Enfermagem) - Fundação Universidade } \\
\text { Federal do Piauí, Teresina. }\end{array}$ & $\begin{array}{l}\text { Avaliar a carga de trabalho de enfermagem em unidade de terapia } \\
\text { intensiva (UTI) por meio da aplicação do Nursing Activities Score (NAS) } \\
\text { e fatores associados à saúde dos trabalhadores dessas unidades. }\end{array}$ \\
\hline D12 & $\begin{array}{l}\text { Martins, A. C. (2011). Sintomas osteomusculares relacionados ao } \\
\text { trabalho de enfermagem em unidade de terapia intensiva. } 142 \text { p. } \\
\text { Dissertação (Mestrado em Gerenciamento em Enfermagem) - } \\
\text { Universidade de São Paulo, São Paulo. }\end{array}$ & $\begin{array}{c}\text { Apreender os sintomas de Distúrbios Osteomusculares Relacionados ao } \\
\text { Trabalho (DORT) entre os trabalhadores de enfermagem, seus } \\
\text { determinantes e possiblidades de prevenção. }\end{array}$ \\
\hline D13 & $\begin{array}{l}\text { Almeida, L. M. N. (2013). Unidade de Terapia Intensiva Neonatal: } \\
\text { riscos físicos como fatores potenciais de agravos à saúde do } \\
\text { trabalhador. } 80 \text { p. Dissertação (Mestrado Profissional em } \\
\text { Bioengenharia) - Universidade do Vale do Paraíba, São José dos } \\
\text { Campos. }\end{array}$ & $\begin{array}{l}\text { Verificar as condições físico-funcionais de uma Unidade de Terapia } \\
\text { Intensiva Neonatal (UTIN) do interior do estado do Maranhão frente às } \\
\text { recomendações da RDC 50/ANVISA/2002 e identificar as fontes } \\
\text { potenciais dos riscos físicos capazes de gerar acidentes de trabalho e } \\
\text { doenças ocupacionais. }\end{array}$ \\
\hline D14 & $\begin{array}{c}\text { Silva, L. C. S. (2013). Percepção dos trabalhadores de saúde sobre a } \\
\text { exposição a micro-organismos multirresistentes. } 110 \text { p. Dissertação } \\
\text { (Mestrado em Enfermagem) - Universidade Federal de Goiás, } \\
\text { Goiânia. }\end{array}$ & $\begin{array}{l}\text { Analisar a percepção de trabalhadores de saúde relacionada à exposição } \\
\text { ocupacional por micro-organismos multirresistentes em uma Unidade de } \\
\text { Terapia Intensiva Infantil de uma instituição do Sistema Único de Saúde } \\
\text { de Goiânia-Goiás. }\end{array}$ \\
\hline D15 & $\begin{array}{c}\text { Benedet, A. (2008). Riscos Físicos: Sua Potencialidade na Unidade } \\
\text { de Terapia Intensiva Neonatal. } 62 \text { p. Dissertação (Mestrado em } \\
\text { Engenharia Biomédica). Universidade do Vale do Paraíba, São José } \\
\text { dos Campos. }\end{array}$ & $\begin{array}{l}\text { Identificar as fontes potenciais dos riscos físicos nas Unidades de Terapia } \\
\text { Intensiva Neonatal de dois hospitais Públicos, um do interior paulista e } \\
\text { outro do interior catarinense, que podem causar acidentes de trabalho e/ou } \\
\text { doenças profissionais. }\end{array}$ \\
\hline D16 & $\begin{array}{c}\text { Lima, F. B. (2014). Prazer e sofrimento na prática laboral do } \\
\text { enfermeiro em uma UTI. } 98 \text { p. Dissertação (Mestrado em } \\
\text { Enfermagem) - Universidade Federal do Estado do Rio de Janeiro, } \\
\text { Rio de Janeiro. }\end{array}$ & $\begin{array}{l}\text { Identificar os fatores que despertam prazer e os que causam sofrimento ao } \\
\text { enfermeiro de uma UTI em sua rotina laboral e discutir como esses } \\
\text { sentimentos de prazer e sofrimento refletem no processo saúde-doença e } \\
\text { trabalho desses enfermeiros, na perspectiva da saúde do trabalhador. }\end{array}$ \\
\hline D17 & $\begin{array}{l}\text { Ramos, E. L. (2009). A qualidade de vida no trabalho: dimensões e } \\
\text { repercussões na saúde do trabalhador de enfermagem de terapia } \\
\text { intensiva. } 117 \text { p. Dissertação (Mestrado em Enfermagem) - } \\
\text { Universidade do Estado do Rio de Janeiro, Rio de Janeiro. }\end{array}$ & $\begin{array}{l}\text { Discutir a qualidade de vida no trabalho do trabalhador de enfermagem de } \\
\text { terapia intensiva; Caracterizar as situações que interferem ou favorecem } \\
\text { na qualidade de vida no trabalho de enfermagem em terapia intensiva e } \\
\text { analisar as repercussões da qualidade de vida no trabalho na saúde do } \\
\text { trabalhador de enfermagem de terapia intensiva. }\end{array}$ \\
\hline D18 & $\begin{array}{c}\text { Santos, C. E. S. (2017). Análise dos riscos ocupacionais dos } \\
\text { profissionais de enfermagem em unidade de terapia intensiva adulto } \\
\text { de um hospital público em imperatriz - MA. } 99 \text { p. Dissertação } \\
\text { (Mestrado em Ciências Ambientais e Saúde) - Pontifícia Universidade } \\
\text { Católica de Goiás, Goiânia. }\end{array}$ & $\begin{array}{l}\text { Analisar os riscos ocupacionais aos quais estão expostos os profissionais } \\
\text { de enfermagem, em Unidade de Terapia Intensiva adulto de um hospital } \\
\text { público de Imperatriz - MA. }\end{array}$ \\
\hline D19 & $\begin{array}{c}\text { Silveira, M. (2014). As repercussões do trabalho noturno para os } \\
\text { trabalhadores de enfermagem de unidades de cuidados intensivos. } \\
111 \text { p. Dissertação (Mestrado em ENFERMAGEM) - Universidade } \\
\text { Federal de Santa Maria, Santa Maria. } \\
\end{array}$ & $\begin{array}{l}\text { Conhecer as repercussões do trabalho noturno aos trabalhadores de } \\
\text { enfermagem de unidades de cuidados intensivos. }\end{array}$ \\
\hline D20 & $\begin{array}{l}\text { Tito, R. S. (2013). Burnout e transtornos mentais comuns nos } \\
\text { trabalhadores de enfermagem que assistem crianças com cadiopatia } \\
\text { grave. } 172 \text { p. Dissertação (Mestrado em Gerenciamento em } \\
\text { Enfermagem) - Universidade de São Paulo, São Paulo. }\end{array}$ & $\begin{array}{l}\text { Identificar a ocorrência de Transtornos Mentais Comuns (TMC), a } \\
\text { ocorrência da síndrome de Burnout, e a associação de ambos os } \\
\text { transtornos nos trabalhadores de enfermagem, bem como elaborar } \\
\text { propostas de intervenção para redução do desgaste psíquico. }\end{array}$ \\
\hline D21 & $\begin{array}{l}\text { Junior, E. F. P. (2012). Inovações tecnológicas em terapia intensiva } \\
\text { repercussões para a saúde do trabalhador de enfermagem e o } \\
\text { processo de trabalho. } 112 \text { p. Dissertação (Mestrado em Enfermagem) } \\
\text { - Universidade do Estado do Rio de Janeiro, Rio de Janeiro. }\end{array}$ & $\begin{array}{c}\text { Identificar a percepção do trabalhador de enfermagem sobre a utilização } \\
\text { de tecnologia dura em Unidade de Terapia Intensiva (UTI); Descrever os } \\
\text { fatores intervenientes em relação ao uso da tecnologia dura pelo } \\
\text { trabalhador de enfermagem de UTI e analisar as repercussões da } \\
\text { utilização da tecnologia dura para o processo de trabalho e a saúde do } \\
\text { trabalhador de enfermagem em UTI. }\end{array}$ \\
\hline D22 & $\begin{array}{c}\text { Rodrigues, M. C. (2017). Riscos ambientais no CTI: um estudo sobre } \\
\text { suas consequências nos profissionais de enfermagem. } 89 \text { p. } \\
\text { Dissertação (Mestrado Profissional em Saúde e Tecnologia no Espaço } \\
\text { Hospitalar) - Universidade Federal do Estado do Rio de Janeiro, Rio } \\
\text { de Janeiro. }\end{array}$ & $\begin{array}{c}\text { Descrever os Riscos Ambientais presentes no CTI caracterizando tipo, } \\
\text { nível e origem; Identificar respostas nos corpos dos profissionais a partir } \\
\text { de como e por que esses riscos a que são submetidos podem causar } \\
\text { adoecimentos; Elaborar indicadores de Riscos Ambientais para os } \\
\text { profissionais de enfermagem a partir de suas respostas. }\end{array}$ \\
\hline D23 & $\begin{array}{c}\text { Soares, R. S. (2018). Burnout e fatores associados entre profissionais } \\
\text { de enfermagem de hospital municipal no Rio de Janeiro. } 79 \mathrm{p} . \\
\text { Dissertação (Mestrado em Enfermagem) - Universidade Federal } \\
\text { Fluminense, Niterói. }\end{array}$ & $\begin{array}{l}\text { Descrever a prevalência e possíveis fatores associados à síndrome de } \\
\text { burnout entre profissionais de enfermagem de setores fechados. }\end{array}$ \\
\hline D24 & $\begin{array}{l}\text { Rocha, A. P. F. (2013). O trabalho em situação limite: o labor e a } \\
\text { saúde dos médicos de uma UTI neonatal. } 85 \text { p. Dissertação (Mestrado } \\
\text { em Saúde Pública) - Fundação Oswaldo Cruz, Rio de Janeiro. }\end{array}$ & $\begin{array}{l}\text { Conhecer a perspectiva sobre a saúde e o trabalho de médicos de uma } \\
\text { unidade de terapia intensiva neonatal de um hospital público do estado do } \\
\text { Rio de Janeiro; interpretar o significado do trabalho na vida desses } \\
\text { profissionais, identificando as dificuldades bem como as satisfações } \\
\text { geradas no âmbito laboral. }\end{array}$ \\
\hline D25 & $\begin{array}{c}\text { Oliveira, R. A. (2013). Desafios à vigilância em saúde do } \\
\text { trabalhador: ações de um núcleo de saúde do trabalhador. } 173 \text { p. } \\
\text { Dissertação (Mestrado em Saúde Pública) - Fundação Oswaldo Cruz, } \\
\text { Rio de Janeiro. }\end{array}$ & $\begin{array}{l}\text { Compreender, por meio de uma reflexão sobre a atuação de um Núcleo de } \\
\text { Saúde do Trabalhador a partir de uma Unidade de Terapia Intensiva } \\
\text { Neonatal (UTIN), os desafios à Vigilância em Saúde do Trabalhador. }\end{array}$ \\
\hline D26 & $\begin{array}{c}\text { Marques, J. P. D. (2017). Satisfação no trabalho de trabalhadores } \\
\text { com formação superior que atuam como técnicos de enfermagem. } 111 \\
\text { p. Dissertação (Mestrado em Enfermagem) - Universidade Federal de } \\
\text { Santa Maria, Santa Maria. }\end{array}$ & $\begin{array}{l}\text { Compreender a satisfação no trabalho de técnicos de enfermagem em } \\
\text { unidades de terapia intensiva, com formação superior. }\end{array}$ \\
\hline D27 & $\begin{array}{l}\text { Conceição, M. A. (2010). Percepção de risco biológico entre } \\
\text { trabalhadores de saúde de um hospital público de médio porte em }\end{array}$ & $\begin{array}{l}\text { Levantamento da percepção de risco biológico entre trabalhadores de } \\
\text { saúde de Unidade de Terapia Intensiva de Adultos de um hospital público }\end{array}$ \\
\hline
\end{tabular}




\begin{tabular}{|c|c|c|}
\hline & $\begin{array}{c}\text { Cuiabá, Mato Grosso. } 105 \text { p. Dissertação (Mestrado Profissional em } \\
\text { Saúde Coletiva) - Faculdade de Ciências Médicas da Sta. Casa de São } \\
\text { Paulo, São Paulo. }\end{array}$ & de médio porte, na cidade de Cuiabá. \\
\hline D28 & $\begin{array}{c}\text { Oliveira, R. P. (2015). Carga de Trabalho e Absenteísmo da Equipe } \\
\text { de Enfermagem em Unidade de Terapia Intensiva. } 104 \text { p. Dissertação } \\
\text { (Mestrado em Enfermagem Fundamental) - Universidade de São } \\
\text { Paulo/ Ribeirão Preto, São Paulo. }\end{array}$ & $\begin{array}{l}\text { Identificar a carga de trabalho e as taxas de absenteísmo da equipe de } \\
\text { enfermagem de uma Unidade de Terapia Intensiva-Adulto (UTI) de um } \\
\text { hospital de ensino no interior do estado de São Paulo. }\end{array}$ \\
\hline D29 & $\begin{array}{l}\text { Silva, G. J. P. (2014). Danos à saúde relacionados ao trabalho em } \\
\text { fisioterapeutas intensivistas da rede hospitalar de São Luís - MA. } 68 \\
\text { p. Dissertação (Mestrado em Saúde Coletiva) - Universidade Federal } \\
\text { do Maranhão, São Luís. }\end{array}$ & $\begin{array}{l}\text { Avaliar os danos à saúde relacionados ao trabalho em fisioterapeutas } \\
\text { intensivistas da Rede Hospitalar de São Luís / Maranhão. }\end{array}$ \\
\hline D30 & $\begin{array}{c}\text { Silva, C. M. S. E. (2017). Saúde do trabalhador em ambiente com } \\
\text { exposição a material biológico: uma produção tecnológica. } 100 \text { p. } \\
\text { Dissertação (Mestrado Profissional em Enfermagem) - Universidade } \\
\text { Federal Fluminense, Niterói. }\end{array}$ & $\begin{array}{l}\text { Elaborar cartilha educativa acerca de exposição a material biológico e dos } \\
\text { procedimentos preconizados no atendimento no Hospital Federal cenário } \\
\text { do estudo; Descrever os sentimentos envolvidos e as necessidades de } \\
\text { informação da equipe de enfermagem após exposição a material biológico } \\
\text { no atendimento realizado no Hospital Federal cenário do estudo; } \\
\text { Disseminar a informação acerca dos procedimentos envolvidos no } \\
\text { atendimento dos membros da equipe de saúde após exposição a material } \\
\text { biológico e Avaliar os aspectos relativos ao conteúdo estrutura da } \\
\text { cartilha educativa com os membros da equipe de enfermagem do CTI do } \\
\text { Hospital cenário do estudo. }\end{array}$ \\
\hline D31 & $\begin{array}{l}\text { Figueiredo, C. A. R. (2015). A percepção do absenteísmo laboral dos } \\
\text { profissionais de enfermagem de unidades de terapia intensiva em } \\
\text { Porto Velho - Rondônia. } 64 \text { p. Dissertação (Mestrado Profissional em } \\
\text { Ensino em Ciências da Saúde) - Universidade Federal de Rondônia, } \\
\text { Porto Velho. }\end{array}$ & $\begin{array}{c}\text { Analisar a percepção do absenteísmo laboral pelos profissionais de } \\
\text { enfermagem das Unidades de Terapia Intensiva do Hospital de Base Dr. } \\
\text { Ary Pinheiro. }\end{array}$ \\
\hline D32 & $\begin{array}{c}\text { Silva, A. P. B. (2018). Riscos e danos relacionados ao contexto do } \\
\text { trabalho da equipe de enfermagem de unidades neonatais. } 136 \text { p. } \\
\text { Dissertação (Mestrado em Enfermagem) - Universidade Federal de } \\
\text { Goiás, Goiânia. }\end{array}$ & $\begin{array}{c}\text { Analisar o contexto de trabalho e os danos ocupacionais, na perspectiva } \\
\text { dos profissionais da equipe de enfermagem, em uma Unidade de Terapia } \\
\text { Intensiva e Intermediaria Neonatal. }\end{array}$ \\
\hline D33 & $\begin{array}{l}\text { Rodrigues, C. M. A. (2016). Sintomas osteomusculares relacionados } \\
\text { ao trabalho de enfermagem em uma unidade de terapia intensiva: } \\
\text { uma abordagem sobre LER/DORT. } 122 \text { p. Dissertação (Mestrado em } \\
\text { Enfermagem) - Universidade Federal do Estado do Rio de Janeiro, } \\
\text { Rio de Janeiro. }\end{array}$ & $\begin{array}{c}\text { Identificar sintomas musculoesqueléticos em trabalhadores de } \\
\text { enfermagem que atuam em uma Unidade de Terapia Intensiva de um } \\
\text { hospital Federal do Estado do Rio de Janeiro e discutir os sintomas } \\
\text { musculoesqueléticos e seus aspectos ergonômicos com ênfase na saúde } \\
\text { do trabalhador. }\end{array}$ \\
\hline T3 & $\begin{array}{l}\text { Fogaça, M. C. (2008). Estresse ocupacional, saúde mental e } \\
\text { qualidade de vida de médicos e enfermeiros intensivistas pediátricos e } \\
\text { neonatais. } 131 \text { p. Tese (Doutorado em Psiquiatria e Psicologia } \\
\text { Médica) - Universidade Federal de São Paulo, São Paulo. }\end{array}$ & $\begin{array}{l}\text { Avaliar o estresse ocupacional, a saúde mental e a qualidade de vida de } \\
\text { médicos e enfermeiros intensivistas pediátricos e neonatais. }\end{array}$ \\
\hline
\end{tabular}

Fonte: Autores.

No que se refere as regiões de publicação das teses e dissertações, 14 produções foram do Rio de Janeiro, 12 do estado de São Paulo, quatro de Goiás e duas do Rio Grande do Sul. Os estados de Santa Catarina, Piauí, Maranhão e Rondônia contaram com um estudo cada. Os programas de pós-graduação em nível de mestrado acadêmico em enfermagem apresentaram um total de 15 dissertações, sendo que destas, 10 são de programas da região sudeste do Brasil. Em relação aos períodos de publicação, entre os anos de 2005 e 2011 foram publicados nove estudos. A maior parte dos estudos foi publicada entre 2012 e 2018, contabilizando 27 produções.

Quanto ao tipo de delineamento do método de pesquisa, 16 produções foram qualitativas (D2, D6, D7, D9, D10, D14, D16, D17, D19, D21, D24, D25, D26, D27, D30, T2) 15 foram quantitativas (D1, D3, D5, D8, D11, D13, D15, D18, D23, D28, D29, D31, D33, T1, T3) e cinco utilizaram a abordagem quanti-qualitativa (D4, D12, D20, D22, D32).

As produções tiveram como participantes, em sua maioria, trabalhadores da equipe de saúde de unidades de terapia intensiva. Desse modo, 21 estudos (D1, D2, D6, D7, D8, D10, D11, D12, D17, D18, D19, D20, D21, D22, D23, D30, D31, D32, D33, T1, T2) investigaram somente a equipe de enfermagem (enfermeiros, técnicos e auxiliares de enfermagem). Além desses, dois estudos (D3, D16) foram realizados somente com enfermeiros, e um estudo ${ }^{(\mathrm{D} 26)}$ teve como participantes técnicos de enfermagem. Cinco estudos ${ }^{(\mathrm{D} 9, \text { D24, }}$ D27, D29, T3) abordaram outros trabalhadores da equipe de saúde além dos de enfermagem, e tiveram como participantes psicólogos, médicos e fisioterapeutas. Destaca-se que quatro estudos (D4, D5, D14, D 25) não especificaram quais trabalhadores de saúde foram investigados. De acordo com os resumos analisados, dois estudos ${ }^{(\mathrm{D} 13, \text { D15) }}{ }^{2}$ utilizaram mapeamento da estrutura física das unidades de terapia intensiva, e um estudo ${ }^{(\mathrm{D} 28)}$ também teve como participantes pacientes internados nesse tipo de unidade. 
Sobre as unidades de terapia intensiva (UTI) que foram cenário das investigações das publicações, sete (D5, D16, D18, D27, D28, D29, T2) foram realizadas em UTI adulto, sete (D4, D10, D13, D15, D24, D25, D32) ocorreram em UTI neonatal, três ${ }^{(\mathrm{D} 7, \mathrm{D} 20, \mathrm{~T} 3)}$ foram desenvolvidas em UTI neonatal e pediátrica, e oito( ${ }^{(\mathrm{D} 6, \mathrm{D} 9, \mathrm{D} 11, \mathrm{D} 12, \mathrm{D} 14, \mathrm{D} 17, \mathrm{D} 19, \mathrm{D} 26)}$ estudos foram realizados em outros tipos especializados de UTI. Verificou-se mediante a análise dos resumos, que 11 (D1, D2, D3, D8, D21, D22, D23, D30, D31, D33, T1) estudos não especificaram quais foram os tipos de UTI investigados.

Em relação as temáticas dos estudos, foram abordados diferentes aspectos acerca da saúde do trabalhador em unidade de terapia intensiva, enfocando assuntos como: trabalho noturno ${ }^{(\mathrm{D} 19, \mathrm{D} 6)}$; carga de trabalho ${ }^{(\mathrm{D} 11, \mathrm{D} 28)}$; riscos de adoecimento para os trabalhadores ${ }^{(\mathrm{D} 3, \mathrm{T1}, \mathrm{D} 8)}$; riscos ocupacionais $^{(\mathrm{D} 18, \mathrm{D} 22)}$; danos ocupacionais ${ }^{(\mathrm{D} 29, \mathrm{D} 32,)}$; riscos do ambiente relacionados a estrutura das unidades ${ }^{(\mathrm{D} 13, \mathrm{D} 15)}$; sintomas osteomusculares ${ }^{(\mathrm{D} 1, \mathrm{D} 12, \mathrm{D} 33)}$; análise postural dos trabalhadores ${ }^{(\mathrm{D} 10)}$; biossegurança $^{(\mathrm{D} 2, \mathrm{D} 14)}$; riscos biológicos $^{(\mathrm{D} 27, \mathrm{D} 30)}$; estresse ${ }^{(\mathrm{D} 9, \mathrm{T3})}$; Burnout ${ }^{(\mathrm{D} 20, \mathrm{D} 23)}$; prazer e sofrimento no trabalho ${ }^{(\mathrm{D} 16)}$; satisfação no trabalho $^{(\mathrm{D} 24, \text { D26)}}$; qualidade de vida no trabalho ${ }^{(\mathrm{D} 17)}$; absenteísmo e presenteísmo ${ }^{(\mathrm{D} 4, \mathrm{D} 5, \mathrm{D} 31)}$; relação entre trabalho e saúde ${ }^{(\mathrm{D} 7, \mathrm{~T} 2)}$; inovações tecnológicas e repercussões para a saúde ${ }^{(\mathrm{D} 21)}$; e vigilância em saúde do trabalhador ${ }^{(\mathrm{D} 25)}$.

\section{Discussão}

Os resultados evidenciaram que a maior parte dos estudos sobre saúde do trabalhador em unidades de terapia intensiva, proveniente de cursos de mestrado acadêmico em enfermagem, é da região sudeste do país. Esse fato vai ao encontro dos dados do último relatório de avaliação quadrienal publicado pela Coordenação de Aperfeiçoamento de Pessoal de Nível Superior (CAPES) referente a área da pós-graduação em enfermagem. No documento, a região sudeste permanece sediando 42,9\% dos programas da área, entre doutorados, mestrados acadêmicos e profissionais (Brasil, 2017).

Os dados referentes ao período de maior número de publicações das produções (27 estudos entre 2012 e 2018) podem estar relacionados ao contexto de proteção legal dos trabalhadores. Em 23 de agosto de 2012, foi instituída a Política Nacional de Saúde do Trabalhador e da Trabalhadora. Esta se configura como conjunto de políticas no domínio do Sistema Único de Saúde (SUS), que consideram a particularidade das ações de saúde do trabalhador, bem como o trabalho como um dos fatores decisivos do processo saúde-doença (Brasil, 2012).

Ainda nesse sentido, destaca-se que a relação do trabalho-saúde vem sendo priorizada nas pesquisas em saúde desde o ano de 2008, com a publicação pelo Ministério da Saúde da subagenda "Saúde, ambiente, trabalho e biossegurança" (Brasil, 2008). Atualmente, a publicação da Agenda de Prioridades de Pesquisa do Ministério da Saúde permanece expondo a necessidade de avaliação de impactos relativos a adoecimentos e agravos relacionados ao trabalho no primeiro eixo temático "Ambiente, trabalho e saúde" (Brasil, 2018).

Nos tipos de método de pesquisa dos estudos analisados, prevaleceram as abordagens qualitativas (D2, D6, D7, D9, D10, D14, D16, D17, D19, D21, D24, D25, D26, D27, D30, T2). O interesse desse tipo de pesquisa está na descrição dos significados das ações e relações para os sujeitos, em convergência com os objetivos propostos. Nesse sentido, busca-se compreender e interpretar principalmente as experiências subjetivas e fenômenos (Minayo, 2014).

Também constatou-se a utilização das abordagens quantitativas (D1, D3, D5, D8, D11, D13, D15, D18, D23, D28, D29, D31, D33, T1, T3) e, em menor número, as quanti-qualitativas ${ }^{(\mathrm{D} 4, \mathrm{D} 12, \mathrm{D} 20, \mathrm{D} 22, \mathrm{D} 32)}$. É relevante enfatizar que os diferentes métodos de investigação tem sua importância e podem se complementar para atender adequadamente as proposições das investigações (Minayo, 2014). Especificamente na área da saúde, essa interação entre as abordagens quanti-qualitativas tornam possíveis avanços para compreender as questões de interesse investigadas (Minayo, 2014).

Os resultados demonstraram que a maior parte dos estudos foi realizada com trabalhadores da equipe de enfermagem como participantes (D1, D3, D2, D6, D7, D8, D10, D11, D12, D16, D17, D18, D19, D20, D21, D22, D23, D26, D30, D31, D32, D33, T1, T2), sendo eles enfermeiros, técnicos e auxiliares de enfermagem. Esse dado corrobora a realidade dos serviços de terapia intensiva nos quais os 
trabalhadores de enfermagem compõe o maior contingente de profissionais de saúde (Souza, et al, 2018). O trabalho da enfermagem é característico por ser mais próximo ao paciente, realizando procedimentos técnicos, cuidados intensivos e assistência permanente ao paciente crítico (Araújo, 2015). Além disso, entende-se que outras condições do trabalho em enfermagem, como sobrecarga de trabalho, desvalorização profissional e déficit de recursos humanos (Oliveira, et al, 2018) tenham interferência na saúde desses trabalhadores.

Ainda em relação aos participantes das produções analisadas, alguns estudos (D9, D24, D27, D29, T3) investigaram outros trabalhadores de saúde que atuam nas unidades de terapia intensiva, como psicólogos, médicos e fisioterapeutas. Pesquisas recentes com médicos intensivistas apontam que estes podem apresentar riscos de desestabilização psicológica, sofrendo por vezes com questões de desgaste emocional e físico (Cherer, Quintana, \& Pinheiro, 2015; Tironi, 2016).

Quanto aos cenários de investigação das teses e dissertações, destacaram-se unidades de terapia intensiva (UTI) destinadas ao atendimento de pacientes adultos ${ }^{(\mathrm{D} 55, \mathrm{D} 16, \mathrm{D} 18, \mathrm{D} 27, \mathrm{D} 28, \mathrm{D} 29, \mathrm{~T} 2)}$ e de assistência neonatal e/ou pediátrica ${ }^{(\mathrm{D} 4, \mathrm{D} 7, \mathrm{D} 10, \mathrm{D} 13,}$ D15, D20, D24, D25, D32, T3). Como já mencionado anteriormente, as UTI são setores diferenciados de complexidade elevada, tecnologias avançadas, pacientes instáveis e assistência especializada (Santos, et al, 2018).

A Resolução número sete de 24 de fevereiro de 2010, da Agência Nacional de Vigilância Sanitária (ANVISA), ainda estabelece algumas padronizações para o funcionamento das UTI: a UTI- Adulto (UTI-A) se destina a pacientes com 18 anos ou mais, podendo admitir pacientes de 15 a 17 anos, se definido pela instituição; a UTI Neonatal (UTI-N) atende recémnascidos (idade entre 0 e 28 dias); a UTI Pediátrica (UTI-P) presta assistência a pacientes com idade entre 29 dias a 14 ou 18 anos, sendo o limite de idade definido conforme cada instituição (Anvisa, 2010).

Dentre estas, provavelmente a que mais se diferencia por suas especificidades seja a UTI neonatal. Esta se caracteriza pelo atendimento de recém-nascidos que necessitam de assistência integral e diferenciada em função de complicações, principalmente prematuridade, com vistas à manutenção das condições de vida e o acompanhamento necessário para sua recuperação (Coelho, 2018).

Dentre os temas dos estudos apresentados no resultados, acerca da saúde do trabalhador em unidades de terapia intensiva, está o trabalho noturno. Em duas dissertações ${ }^{(\mathrm{D} 6, \mathrm{D} 19)}$, esse assunto foi pesquisado em equipes de trabalhadores de enfermagem. Ambos os estudos evidenciaram que o trabalho noturno pode ter repercussões para saúde dessa população, com alterações psicofisiológicas $^{(\mathrm{D} 6)}$ e interferência na vida social, familiar e emocional ${ }^{(\mathrm{D} 19)}$.

$\mathrm{O}$ trabalho de enfermagem em setor hospitalar realmente tem a característica de ser desenvolvido em turnos. Trabalhadores de enfermagem que realizam plantões noturnos geralmente tem atendidas as necessidades relacionadas a aspectos financeiros e conciliação com mais de um emprego (Fernandes, et al, 2017). Porém, atuar no período noturno implica na inversão do funcionamento fisiológico dos estados de descanso e vigília, levando distúrbios psíquicos, cardiovasculares e gastrintestinais (Fernandes, et al, 2017).

A carga de trabalho também foi tema de duas dissertações ${ }^{(\mathrm{D} 11, \text { D28) }}{ }^{2}$ realizadas com a aplicação do instrumento NAS (Nursing Activities Score). Nos dois estudos a demanda de trabalho foi elevada nas UTI, com alta média do NAS, refletindo sobrecarga para os trabalhadores de enfermagem ${ }^{(\mathrm{D} 11)}$ e necessidade de reflexão sobre o adequado dimensionamento de pessoal nesses setores ${ }^{(\mathrm{D} 28)}$. Estudo que utilizou o mesmo instrumento de avaliação também enfatiza a necessidade de prover condições de trabalho, inferindo a importância da adequação do quantitativo de trabalhadores de enfermagem, bem de sua qualificação para o cuidado adequado em UTI (Souza, 2019).

Três estudos abordaram riscos de adoecimento para trabalhadores de enfermagem ${ }^{(\mathrm{D} 3, \mathrm{D} 8, \mathrm{~T} 1)}$. Uma dissertação ${ }^{(\mathrm{D} 3)}$ evidenciou especificamente o risco de adoecimento do profissional enfermeiro que atua em UTI. Outra dissertação ${ }^{\left({ }^{(D 8)}\right.}$ com 43 trabalhadores de enfermagem indicou que 72,09\% referiram queixas de saúde, sendo as mais frequentes as doenças do sistema 
osteomuscular e tecido conjuntivo $(\mathrm{N}=18 ; 32,73 \%)$. Ainda nesse sentido, uma tese ${ }^{(\mathrm{T} 1)}$ abordou as repercussões do ruído ambiental para trabalhadores de enfermagem, constatando alterações de frequência cardíaca e pressão arterial.

Nesse contexto, duas dissertações (D18, D22) contemplaram em suas investigações com trabalhadores de enfermagem a questão dos riscos ocupacionais da UTI. Demonstraram que nesse ambiente os trabalhadores estão expostos a diferentes tipos de riscos, como os de acidentes, químicos ${ }^{(\mathrm{D} 18)}$, ergonômicos ${ }^{(\mathrm{D} 18, \mathrm{D} 22)}$, físicos, biológicos e psicossociais ${ }^{(\mathrm{D} 22)}$.

Ainda em relação aos riscos, duas dissertações apresentaram o mapeamento de riscos do ambiente relacionados a estrutura física de UTIs neonatais (D13, D15). Os resultados apontaram a existência riscos em função da composição estrutural, como pisos sem proteção antiderrapante, disposição inadequada de mobiliário e equipamentos, instalações elétricas inadequadas, iluminação insuficiente, ruído, calor e exposição a radiações ${ }^{(\mathrm{D} 13)}$, podendo causar acidentes de trabalho ${ }^{(\mathrm{D} 15)}$.

Outras duas dissertações ${ }^{(\mathrm{D} 29, \text { D32) }}$ discorreram sobre danos ocupacionais para a saúde. Uma dissertação ${ }^{(\mathrm{D} 29)}$ teve como participantes fisioterapeutas intensivistas, constatando a frequência de danos osteomusculares e respiratórios para estes trabalhadores. A outra dissertação ${ }^{(\mathrm{D} 32)}$ foi realizada com trabalhadores de enfermagem de UTI neonatal, e constatou que o contexto laboral dessas unidades predispõe a danos físicos, psíquicos e sociais.

Duas dissertações ( ${ }^{\mathrm{D} 27, \mathrm{D} 30)}$ abordaram os riscos biológicos presentes em UTI. Uma delas ${ }^{(\mathrm{D} 27)}$, realizada com médicos, fisioterapeutas, enfermeiros, auxiliares e técnicos de enfermagem, observou a exposição desses trabalhadores a agentes biológicos. A outra dissertação ${ }^{(\mathrm{D} 30)}$, que teve como participantes trabalhadores de enfermagem, revelaram a insegurança que estes sentem em relação a possibilidade de contaminação com materiais biológicos e o desenvolvimento de doenças.

Nesse contexto, cada trabalhador da equipe de saúde deve estar atento aos riscos ocupacionais aos quais está exposto, com vistas a sua própria proteção (Ribeiro, et al, 2016). Esses riscos podem ser entendidos como ações ou situações no seu espaço laboral que podem comprometer a saúde do trabalhador (Miguel et al, 2014). Em uma definição mais abrangente, o termo expressa que os riscos ocupacionais são todas e quaisquer possibilidades de ocorrência de acidente, dano, adoecimento ou sofrimento ao trabalhador devido a algum fator presente no processo de trabalho (Porto, 2000).

Dentre os tipos de riscos citados nas produções, conceitua-se suas definições: os riscos físicos se configuram como a exposição a agentes físicos, como ruídos, radiação e até mesmo a temperatura do ambiente; os riscos químicos se caracterizam principalmente pela exposição aos medicamentos, produtos químicos e gases ou vapores; os riscos biológicos estão associados a exposição a microrganismos, secreções e patógenos em geral; o risco ergonômico se refere as questões que podem desencadear lesões osteomusculares e também as que podem levar a desestabilização de cunho psicológico do profissional (Brasil, 2001).

$\mathrm{O}$ tema biossegurança, foi discutido em duas dissertações ${ }^{(\mathrm{D} 2, \mathrm{D} 14)}$. Uma delas ${ }^{(\mathrm{D} 2)}$, que teve como participantes a equipe de enfermagem, concluiu que a adoção e implementação das medidas de biossegurança na UTI se restringiram a utilização dos equipamentos de proteção individual (EPI), lavagem das mãos e cuidados com perfurocortantes. Outra dissertação ${ }^{(\mathrm{D} 14)}$, que analisou a percepção de trabalhadores da equipe multiprofissional de UTI relacionada à exposição ocupacional por microorganismos multirresistentes, evidenciou que os trabalhadores não têm clareza sobre comportamentos relacionados a segurança laboral. A Lei número 11.105, de 24 de março de 2005 discorre sobre a biossegurança, e estabelece normas e mecanismos de fiscalização de atividades que envolvam organismos geneticamente modificados - OGM e seus derivados. Essa lei também cria o Conselho Nacional de Biossegurança - CNBS, reestrutura a Comissão Técnica Nacional de Biossegurança- CTNBio, e dispõe sobre a Política Nacional de Biossegurança - PNB (Brasil, 2005). Destaca-se que a implementação e cumprimento de normas de biossegurança se fazem essenciais para a segurança dos trabalhadores de todas as áreas (Paredes et al, 2013).

Sobre sintomas osteomusculares, três dissertações (D1, D12, D33), todas realizadas com trabalhadores de enfermagem, destacaram sintomas lombares ${ }^{(\mathrm{D} 1)}$ e sintomas musculoesqueléticos na região das costas ${ }^{(\mathrm{D} 12, \mathrm{D} 33)}$. Nos três estudos, as condições do trabalho em UTI, como repetitividade, falta de descanso, manipulação de peso excessivo, desajustes na estrutura física, 
déficit de recursos humanos, sobrecarga, foram associados como contribuintes para o desenvolvimento dos sintomas no trabalhadores (D1, D12, D33). Outros trabalhadores de saúde que prestam assistência direta ao paciente também podem apresentar tais condições. Investigação retrospectiva com fisioterapeutas de um hospital universitário, mostrou que após um ano atuando em UTI a quantidade de autorrelato de sintomas de dor e desconforto musculoesquelético aumentou, quando comparada a outros setores da instituição (Santos, et al, 2018).

Outra dissertação ${ }^{(\mathrm{D} 10)}$ analisou a postura de trabalhadoras de enfermagem durante o banho do recém-nascido em UTI neonatal. Identificou que a altura e organização dos materiais na unidade influenciam nas posturas adotadas pelo trabalhador, causando grande sobrecarga muscular devido ao trabalho estático sofrido, que podem gerar fadiga e danos a musculatura ${ }^{(\mathrm{D} 10)}$.

Habitualmente os pequenos pacientes da UTI neonatal estão acomodados em berço aquecido ou incubadora, equipamentos estes que provem calor elevado ao bebê que ainda não consegue estabelecer sua própria regulação térmica (Cardoso, et al, 2015) e, geralmente, não possuem regulação de altura em relação ao trabalhador. Do mesmo modo, esses mesmos equipamentos produzem sinalizações sonoras, além de outros equipamentos de uso comum que ficam próximos aos leitos e geram ruídos, como ventiladores mecânicos e monitores (Cardoso, et al, 2015).

O estresse ocupacional também foi abordado em duas produções ${ }^{(\mathrm{D} 9, \mathrm{~T} 3)}$. A dissertação ${ }^{(\mathrm{D} 9)}$ foi realizada com psicólogos, enfermeiros, fisioterapeutas, e identificou fatores associados condições ambientais (inadequação da planta física, de temperatura, poluição sonora e carga de trabalho) como fontes de estresse importantes gerando desgaste físico e emocional. A tese $^{(\mathrm{T} 3)}$ teve como participantes médicos e enfermeiros de UTI pediátrica e neonatal, e apresentaram altas demandas psicológicas que repercutiram na qualidade de vida no trabalho. Estudos realizados com médicos intensivistas corroboram esses dados, enfatizando que estes podem sofrer com questões emocionais e psicológicas (Tironi, 2016), bem como tensão pelo medo da perda dos pacientes (Rocha, Souza, \& Teixeira, 2015).

Uma dissertação ${ }^{(\mathrm{D} 21)}$ com trabalhadores de enfermagem sobre inovações tecnológicas e repercussões para a saúde destacou que o uso das tecnologias duras em UTI possibilitam maior segurança e controle sobre o estado clínico dos pacientes, porém, problemas na manutenção dos aparelhos acarretam sobrecarga mental e física devido a necessidade constante de ajustes, e estes fatores podem gerar risco psicossocial por acarretarem estresse ocupacional.

Seguindo as questões de saúde mental relacionadas ao trabalho, outras dissertações também abordaram o tema. A síndrome de Burnout foi discutida em duas dissertaçoes (D20, D23), que tiveram como participantes trabalhadores de enfermagem, sendo que ambas constataram escores elevados para suspeita da condição nesses trabalhadores, considerando dimensões de avaliação como esgotamento emocional, por exemplo. Destaca-se que estudos com outros trabalhadores da equipe de saúde também verificam a ocorrência da síndrome. Estudo com fisioterapeutas intensivistas apontou elevada prevalência de Burnout nesses trabalhadores, sendo a de exaustão emocional também foi a que se destacou (Santos, et al, 2018). Do mesmo modo, pesquisa com médicos intensivistas de cinco capitais do Brasil evidenciou que a dimensão de exaustão emocional foi a que mais contribuiu no resultado elevado para a síndrome (Tironi, et al, 2016).

Nesse sentido, uma dissertação ${ }^{(\mathrm{D} 16)}$ abordou questões de prazer e sofrimento no trabalho de enfermeiros de UTI, identificando que algumas condições ambientais, estruturais e funcionais estão relacionadas ao sofrimento destes trabalhadores em seu ambiente laboral.

Outras dissertações (D24, D26) investigaram aspectos da satisfação no trabalho. Uma delas ${ }^{(\mathrm{D} 24)}$ realizada com médicos de UTI neonatal concluiu que condições inadequadas de trabalho geram desestabilização na saúde desses trabalhadores e geram riscos para manutenção da atividade laboral de qualidade. Outra dissertação ${ }^{(\mathrm{D} 26)}$, na qual participaram trabalhadores que atuam como técnicos de enfermagem mas tem formação superior, identificou, dentre outros pontos, que, mesmo considerando positivo ter um conhecimento mais aprofundado, estes trabalhadores sentem frustração por não poderem atuar desenvolvendo atividades aprendidas no curso superior. Ainda nesse contexto, uma dissertação ${ }^{(\mathrm{D} 17)}$ abordou a qualidade de vida no trabalho 
em trabalhadores de enfermagem, considerando que as cargas de trabalho provocam desgaste físico e mental, gerando desestimulo em relação ao trabalho e sofrimento psíquico.

Três dissertações (D4, D5, D31) abrangeram os temas de presenteísmo e absenteísmo. Sobre o presenteísmo, duas produções (D4, D5) realizadas com equipe de trabalhadores de saúde verificaram que estes comparecem ao trabalho mesmo estando doentes, o que pode comprometer ainda mais o seu estado físico e mental. Uma dissertação ${ }^{(\mathrm{D} 31)}$ destacou o absenteísmo em trabalhadores de enfermagem, tendo como fatores de relevância para falta ao trabalho a baixa remuneração, o cansaço, a sobrecarga e as condições laborais deficientes.

Presenteísmo se configura como a presença física do trabalhador no seu trabalho, porém em estado de desconexão em relação ao comprometimento necessário para desenvolver suas atividades de forma eficaz devido a questões de ordem física ou psicológica (Camargo, 2017). O absenteísmo é entendido como a falta ao trabalho e se constituí como importante ponto de avaliação das condições de saúde dos trabalhadores, recursos humanos e satisfação com o trabalho (Heylmann, et al, 2016).

Desse modo, pensando no contexto da interferência do trabalho sobre a saúde, duas produções ${ }^{(D 7, T 2)}$ evidenciaram essas relações especificamente com trabalhadores da equipe de enfermagem de terapia intensiva. Apontaram que condições materiais e organizacionais desafiam a manutenção da saúde e geram desgaste para esses trabalhadores. Nesse sentido, uma dissertação ${ }^{(\mathrm{D} 25)}$ enfatizou a importância da consolidação das ações de vigilância em saúde do trabalhador em setores hospitalares.

A proteção do trabalhador de saúde conta com uma legislação própria. Em vigor desde a aprovação da Portaria número 485 de novembro de 2005, a Norma Regulamentadora número 32 (NR) estabelece diretrizes fundamentais para implantação de medidas que promovam a segurança e assegurem assistência à saúde dos trabalhadores de serviços de saúde. A NR 32 conceitua como serviço de saúde todo estabelecimento onde é prestada assistência à saúde em qualquer nível de complexidade, incluindo o desenvolvimento de processos de pesquisa e ensino em saúde (Brasil, 2005).

\section{Considerações Finais}

Com a realização do presente estudo foi possível identificar as tendências da produção científica, em teses e dissertações, sobre saúde do trabalhador em unidades de terapia intensiva. A maior parte das produções foram dissertações provenientes de cursos de mestrado em enfermagem, da região sudeste do Brasil e após o ano de 2012. As pesquisas foram predominantemente realizadas com trabalhadores da equipe de enfermagem, abrangendo os diversos tipos de UTI, com destaque para UTI neonatal, pediátrica e adulta.

Dentre as temáticas encontradas, estiveram destacadas nas produções as características próprias do trabalho em terapia intensiva e das profissões da área da saúde, os riscos ocupacionais que estão presentes no cotidiano das UTI, bem como os diversos e preocupantes acometimentos que podem vir a trazer prejuízos para a saúde desses trabalhadores.

É importante enfatizar que nas dissertações e teses analisadas ficou evidente a constante relação entre a influência das atividades laborais sobre a saúde dos trabalhadores. Assim, se demonstra a necessidade permanente e a relevância de investigações acerca da saúde do trabalhador em unidades de terapia intensiva, com vistas a esclarecer e proporcionar visibilidade para essa complexa problemática da atualidade. Sugere-se, desse modo, que novos estudos e pesquisas com diferentes abordagens metodológicas sejam desenvolvidos abarcando e contribuindo com tais temáticas.

\section{Referências}

Anvisa. (2010). Resolução ANVISA/DC No 7, de 24 de fevereiro de 2010. Dispõe sobre os requisitos mínimos para funcionamento de Unidades de Terapia Intensiva e dá outras providências. (ANVISA). Diário Oficial da União; Poder Executivo, Brasília, DF, I, p. 48-51.

Araújo, S. N. P. (2015). Os riscos enfrentados pelos profissionais de enfermagem no exercício da atividade laboral. Rev Enferm Contemporânea, 4(2), 237-43. 
Brasil. (2005). Lei n. 11.105, de 25 de março de 2005.

Brasil. (2017). Ministério da Educação. Coordenação de Aperfeiçoamento de Pessoal de Nível Superior. Diretoria de Avaliação. Relatório de Avaliação Quadrienal 2017 - Enfermagem.

Brasil. (2001). Ministério da Saúde. Doenças relacionadas ao trabalho: manual de procedimentos para os serviços de saúde. Série A. Normas e Manuais Técnicos; n.114. Brasília.

Brasil. (2012). Ministério da Saúde. Portaria n 1.823, de 23 de agosto de 2012. Institui a Política Nacional de Saúde do Trabalhador e da Trabalhadora. Brasília.

Brasil. (2008). Ministério da Saúde. Secretaria de Ciência, Tecnologia e Insumos Estratégicos. Departamento de Ciência e Tecnologia. Seleção de prioridades de pesquisa em saúde: guia PPSUS. Brasília.

Brasil. (2018). Ministério da Saúde. Secretaria de Ciência, Tecnologia e Insumos Estratégicos. Departamento de Ciência e Tecnologia. Agenda de Prioridades de Pesquisa do Ministério da Saúde - APPMS [recurso eletrônico] Brasília.

Brasil. (2005). Ministério do trabalho e emprego. Portaria n. ${ }^{\circ}$ 485, de 11 de novembro de 2005. Aprova a Norma Regulamentadora n. ${ }^{\circ} 32$ (Segurança e Saúde no Trabalho em Estabelecimentos de Saúde). Brasília.

Camargo, M. L. (2017). Presenteísmo: denúncia do mal-estar nos contextos organizacionais de trabalho e de riscos à saúde do trabalhador. R. Laborativa, 6(1), 125-146.

Cardoso, S. M. S., Kozlowskic, L. C., Lacerdaa, A. B. M., Marquesa, J. M., \& A. Ribasd. (2015). Newborn physiological responses to noise in the neonatal unit. Braz J Otorhinolaryngol, 81(6), 583-588.

Cherer, E. Q., Quintana, A. M., \& Pinheiro, U. M. S. (2015). A Morte na Perspectiva de Enfermeiros e Médicos de uma Unidade de Terapia Intensiva Pediátrica. Estud. psicol., 32(4), 685-694.

Coelho, A. S., Custódio, D. C. G. G., Silva, J. S. C., Rosso, G., Silva, R., \& Carniel, F. (2018). Equipe de Enfermagem e a assistência humanizada na UTI neonatal. Reon Facema, 4(1), 873-877.

Fernandes, B. K. C., Ribeiro, A. C. M., Borges, C. L., Galiza, F. T., \& Joventino, E. S. (2017). Influências do trabalho noturno no sono dos trabalhadores de enfermagem: revisão integrativa. Revista Enfermagem Atual, 81(19), 97-103.

Forte, E. C. N., Trombetta, A. P., Pires, D. E. P., Gelbcke, F. L., \& Lino, M. M. (2014). Abordagens teóricas sobre a saúde do trabalhador de enfermagem: revisão integrativa. Cogitare Enferm, 19(3), 604-11.

Gomez, C. M., Vasconcellos, L. C. F., \& Machado, J. M. H. (2018). Saúde do trabalhador: aspectos históricos, avanços e desafios no Sistema Único de Saúde. Rev. Ciência \& Saúde Coletiva, 23(6), 1963-1970.

Heylmann, N. R., Nunes, J. F. S., Fermino, N., \& Ascari, R. A. (2016). Absenteísmo entre profissionais de enfermagem: estudo num hospital universitário catarinense. Revista UNINGÁ Review, 26(2), 10-15.

Lacerda, M. R. \& Costenaro, R. G. S. (2016). Metodologias da pesquisa para a enfermagem e saúde: da teoria à prática. Moriá.

Miguel, D. B. et al. (2014). Percepção de trabalhadores de uma unidade oncológica acerca dos riscos ocupacionais. Cienc Cuid Saude, 13(3), 527-534.

Minayo, M. C. S. (2014). O desafio do conhecimento: pesquisa qualitativa em saúde. (14a ed). Hucitec,

Oliveira, A. K. S., Fernandes, A. M. G., Carvalho, G. A. F. L., Nascimento, L. K. A. S., Pellense, M. C. S., \& Santana, P. G. C. (2018). Humanização da assistência de enfermagem na unidade de terapia intensiva. Revista Humano Ser - UNIFACEX, 3(1), 128-145.

Paredes, A., Pimentel, M. I. C., Firmo, W. C. A., Pontes, S. R. S., Ferreira, V. S., Lima, T. D. V., \& Lago, R. J. M. (2013). Conhecimento e prática sobre medidas de biossegurança por técnicos em enfermagem em um hospital de referência em oncologia em São Luis, Maranhão, Brasil. J Manag Prim Health Care, 4(2), 87-93.

Porto, M. F. S. (2000). Análise de riscos nos locais de trabalho: conhecer para transformar. Cadernos de Saúde do Trabalhador: Instituto Nacional de Saúde do Trabalhador (INST). Kingraf.

Ribeiro, I. P., Rodrigues, A. M., Silva, I. C., \& Santos, J. D. (2016). Riscos ocupacionais da equipe de enfermagem na hemodiálise. R. Interd, 9(1), $143-152$.

Rocha, A. P. F., Souza, K. R., \& Teixeira, L. R. (2015). A saúde e o trabalho de médicos de UTI neonatal: um estudo em hospital público no Rio de Janeiro. Physis Revista de Saúde Coletiva, 25(3), 843-862.

Santos, C. L. C., Barbosa, G. B., Nascimento, D. S. S., Martins Júnior, D. F., \& Nascimento Sobrinho, C. L. (2018). Prevalência de Síndrome da Estafa Profissional e fatores associados em fisioterapeutas intensivistas. Rev Pesq Fisio, 8(3), 336- 344.

Santos, E. L., Dórea, S. N. A., Maciel, M. P. G. S., Santos, L. K. F., Silva, M. B., \& Moraes, M. G. L. (2018). Assistência humanizada: percepção do enfermeiro intensivista. Rev baiana enferm, 32:e23680.

Santos, R. M. E., Maduro, P. A., Silva, T. F. A., \& Trombini-Souza, F. (2018). Dor e desconforto musculoesquelético em fisioterapeutas da unidade de terapia intensiva e enfermaria de um hospital universitário: um estudo de coorte retrospectivo. Br J Pain, 1(2), 127-33.

Silva, A. F., Robazzi, M. L. C. C., Dalri, R. C. M. B., \& Monteiro, C. A. S. (2019). Absenteísmo na equipe multiprofissional de uma unidade de terapia intensiva adulto. Rev. Expr. Catól. Saúde, 4(1). 
Research, Society and Development, v. 10, n. 5, e28510515004, 2021

(CC BY 4.0) | ISSN 2525-3409 | DOI: http://dx.doi.org/10.33448/rsd-v10i5.15004

Souza, K. A., Paula, V. G., Bridi, A. C., Camerini, F. G., Franco, A. S., \& Pereira, B. S. L. (2018). Condutas dos Profissionais de Enfermagem Frente os Alarmes dos Ventiladores Mecânicos em uma Unidade de Terapia Intensiva. Revista de Enfermagem do Centro-Oeste Mineiro, 8/2678.

Souza, V. S., Oliveira, J. L. C., Costa, M. A. R., Vicente, G., Mendonça, R. R., \& Matsuda, L. M. (2019). Associação entre clima de segurança e a carga de trabalho da enfermagem. Cogitare enferm, 24: e58976.

Tironi, M. O., Teles, J. M. M., Barros, D. S., Vieira, D. F. V. B., Silva Filho, C. M., Martins Júnior, D. F., Matos, M. A., \& Nascimento Sobrinho, C. L. (2016). Prevalência de síndrome de burnout em médicos intensivistas de cinco capitais brasileiras. Rev Bras Ter Intensiva, 28(3), 270-277. 